\title{
An investigation of the structure of ensemble averaged extreme wind events
}

\author{
A. Scarabino ${ }^{\dagger}$ \\ Departamento Aeronautica, Universidad Nacional de La Plata, \\ Calle 116 e/ 47 y 48, 1900 La Plata, Argentina \\ M. Sterling \\ School of Engineering, The University of Birmingham, Edgbaston, Birmingham, B15 2TT, UK \\ P. J. Richards \\ School of Engineering, University of Auckland, New Zealand \\ C. J. Baker and R. P. Hoxey \\ School of Engineering, The University of Birmingham, Edgbaston, Birmingham, B15 2TT, UK \\ (Received September 22, 2006, Accepted January 22, 2007)
}

\begin{abstract}
This paper examines the extreme gust profiles obtained by conditionally sampling full-scale velocity data obtained in the lower part of the atmospheric boundary layer. It is demonstrated that three different types of behaviour can be observed in the streamwise component of velocity. In all cases the corresponding vertical velocity component illustrates similar behaviour. An idealised horseshoe vortex model and a downburst model are investigated to examine if such structures can explain the behaviour observed. In addition, an empirical model is developed for an isolated gust corresponding to each of the three types of behaviour observed. It is possible that the division of the gust profile into three different types may lead to an improvement in the correlation of extreme gust events with respect to type.
\end{abstract}

Keywords: gust shapes; conditional sampling; ensemble averaging; downbursts; hairpin vortex structures.

\section{Introduction}

Exactly what constitutes a vortex and consequently what factors affect the bursting and sweeping events that occur in the atmospheric boundary layer (ABL) are issues that have vexed a great number of people. Numerous definitions and counter definitions exist concerning vortices (Fiedler 1988, Robinson 1991) in addition to a variety of methods which purport to be able to identify such

† Corresponding Author, E-mail: scarabino@ing.unlp.edu.ar 
structures (Hunt, et al. 1988, Chong, et al. 1990, Jeong and Hussian 1995, Cucitore, et al. 1999, Adrian, et al. 2000). In essence these methods essentially involve conditionally sampling the velocity field or a function of the velocity subject to a variety of threshold criteria. Whatever definition/identification method is adopted the resulting output highlight the complex nature of the lower part of the ABL even over smooth rural terrain.

In the UK several attempts have been made to identify such structures albeit with limited success (Sterling, et al. 2005, 2006). Through analysis of velocity and pressure time series obtained in the lower part of the ABL in a rural environment, certain ensemble averaged features have been identified. For example, during extreme events (defined as the streamwise velocity $\geq 99.95^{\text {th }}$ percentile velocity) it has been demonstrated that discrete high energy, high frequency events can be considered to be superimposed on low energy, low frequency structures. This insight has been obtained using a conditional sampling technique which up to now has consistently demonstrated that such structures are asymmetrical in the time domain, i.e., there is a rapid increase in localised velocity prior to the event and a relatively slow decrease in velocity immediately after the event. Such asymmetry has also been reported by Larsen, et al. (2003) who proposed a model which reproduced the pre-event behaviour reasonably well, but unfortunately did not have the same success for the post-event behaviour. The importance of such extreme events in terms of wind loading and the design of low-rise buildings have been demonstrated in Sterling, et al. (2003). The aim of this paper is to re-examine the characteristics of a number of extreme events which have already been analysed in Sterling, et al. (2006), and to illustrate that previous ensemble averaging of such structures may have hidden a number of characteristics and also masked the behaviour of a number of different types of flow characteristics.

Section 2 of the paper outlines the experimental data and reviews some of the important flow phenomena which are relevant to subsequent sections. In some cases the data has been analysed elsewhere (Sterling, et al. 2006) and where this is the case only key figures/data are reported for the benefit of the reader. However, the majority of the data analysis presented in this paper is new and relevant to discussions that subsequently develop. Section 3 of the paper introduces a symmetry parameter which enables the classification of three different types of flow behaviour. The behaviour of these flows are analysed in detail and appropriate conclusions are drawn. It is often assumed that hairpin structures and/or downbursts are responsible for a number of the extreme wind velocities that occur in the lower part of the atmospheric boundary layer. Both of these structures are modelled in Section 4 and the results are compared with those of Section 3. Finally, Section 5 presents some conclusions and discusses the implications of the work.

\section{Experimental data}

In what follows data relating to a series of full-scale experiments recorded in natural wind conditions at the wind engineering site at the former Silsoe Research Institute, Bedford, UK are analysed. The experimental site is well documented (Richards, et al. 1997) and is orientated to face the prevailing WSW winds with a large unobstructed fetch. For all of the experiments four three component ultrasonic anemometers (R3 Research, Gill Instruments, Lymington, UK) were positioned at heights $1 \mathrm{~m}, 3 \mathrm{~m}, 6 \mathrm{~m}$ and $10 \mathrm{~m}$ above the ground and sampled simultaneously at a period of $0.1 \mathrm{~s}$. Twelve one-hour datasets numbered $d s 1-d s 12$ were obtained with similar flow statistics, see Table 1. All experiments were carried out in accordance with normal practice, i.e., the total length of the measuring period was one hour and as such was sufficient to ensure that that the 
Table 1 A summary of the flow statistics obtained from the full-scale experiments

\begin{tabular}{|c|c|c|c|c|c|c|c|c|c|}
\hline \multicolumn{10}{|c|}{ Experiment 1} \\
\hline $\begin{array}{l}\text { Height above } \\
\text { the ground }\end{array}$ & 1 & 3 & 6 & 10 & \multirow{2}{*}{$\begin{array}{c}u_{*} \\
(\mathrm{~m} / \mathrm{s})\end{array}$} & 1 & 3 & 6 & 10 \\
\hline Dataset & \multicolumn{4}{|c|}{$U(\mathrm{~m} / \mathrm{s})$} & & \multicolumn{4}{|c|}{$I_{u}$} \\
\hline$d s 1$ & 7.29 & 9.01 & 9.91 & 10.54 & 0.57 & 0.24 & 0.20 & 0.19 & 0.18 \\
\hline$d s 2$ & 6.93 & 8.61 & 9.46 & 10.06 & 0.54 & 0.24 & 0.20 & 0.19 & 0.18 \\
\hline$d s 3$ & 6.88 & 8.56 & 9.42 & 10.00 & 0.55 & 0.23 & 0.19 & 0.18 & 0.17 \\
\hline$d s 4$ & 6.75 & 8.39 & 9.20 & 9.78 & 0.53 & 0.23 & 0.20 & 0.19 & 0.18 \\
\hline$d s 5$ & 7.21 & 8.92 & 9.87 & 10.46 & 0.56 & 0.24 & 0.21 & 0.19 & 0.19 \\
\hline$d s 6$ & 7.12 & 8.85 & 9.77 & 10.37 & 0.57 & 0.24 & 0.21 & 0.19 & 0.19 \\
\hline$d s 7$ & 7.03 & 8.68 & 9.51 & 10.11 & 0.54 & 0.24 & 0.20 & 0.18 & 0.18 \\
\hline$d s 8$ & 6.86 & 8.50 & 9.36 & 10.00 & 0.55 & 0.25 & 0.22 & 0.20 & 0.20 \\
\hline$d s 9$ & 6.36 & 7.92 & 8.71 & 9.25 & 0.50 & 0.25 & 0.21 & 0.20 & 0.19 \\
\hline$d s 10$ & 7.16 & 8.87 & 9.77 & 10.43 & 0.57 & 0.25 & 0.21 & 0.20 & 0.19 \\
\hline$d s 11$ & 6.97 & 8.70 & 9.62 & 10.25 & 0.57 & 0.26 & 0.22 & 0.21 & 0.20 \\
\hline$d s 12$ & 7.09 & 8.79 & 9.68 & 10.30 & 0.56 & 0.25 & 0.22 & 0.20 & 0.19 \\
\hline Average $=$ & 6.97 & 8.65 & 9.52 & 10.13 & 0.55 & 0.24 & 0.21 & 0.19 & 0.19 \\
\hline Stdev $=$ & 0.25 & 0.29 & 0.33 & 0.36 & 0.02 & 0.01 & 0.01 & 0.01 & 0.01 \\
\hline
\end{tabular}

lower frequencies of the data tend towards those corresponding to little energy in the spectral gap, and the sampling frequency was such that the high frequency components which were not measured also contained relatively little energy.

In Table $1, U$ is the mean streamwise velocity, $u_{*}$ is the friction velocity, $I_{u}$ is the streamwise turbulence intensity $\left(\sigma_{u} / U\right)$, and $\sigma_{u}$ is the standard deviation of the streamwise velocity. Full details of the experimental setup and flow statistics can be found in Sterling, et al. (2006).

In order to examine the behaviour of the velocity components during a peak event a conditional sampling technique described fully in Sterling, et al. (2006) was employed. This is outlined below for completeness. Peak events are defined when the velocity at a particular anemometer exceeds a given value (e.g. the $99.95^{\text {th }}$ percentile). "Events" less than two seconds apart are taken to be one event with the peak value being given by the larger value. Such a method results in a number of mini time series which can then be ensemble averaged. The results of such an approach using the ultrasonic anemometer at $1 \mathrm{~m}$ above the ground are shown in Fig. 1. In Fig. 1, the vertical axis represents the fluctuating velocity component normalised by its standard deviation. Fig. 1(a) relates to the streamwise velocity component $\left(u^{\prime}\right)$ and illustrates a sharp localised peak at the event time. Fig. 1(b) illustrates the behaviour of the lateral velocity component $\left(v^{\prime}\right)$. It is evident that the distribution of this component is relatively uniform across the entire event time. There is also a rather large variation between each of the datasets, but no other distinctive trends are apparent. Fig. 1(c) relates to the vertical component $\left(w^{\prime}\right)$ and possibly illustrates the most interesting behaviour. Prior to the event time $(t=-2.5 \sim-0.1 \mathrm{~s})$ the distributions across all of the datasets are reasonably uniform. However, between $t=-0.1 \mathrm{~s}$ and $t=0 \mathrm{~s}$, a rapid decrease in $w^{\prime} / \sigma_{w}$ is shown in all of the datasets. This suggests that the extreme events are associated with a so called sweeping 


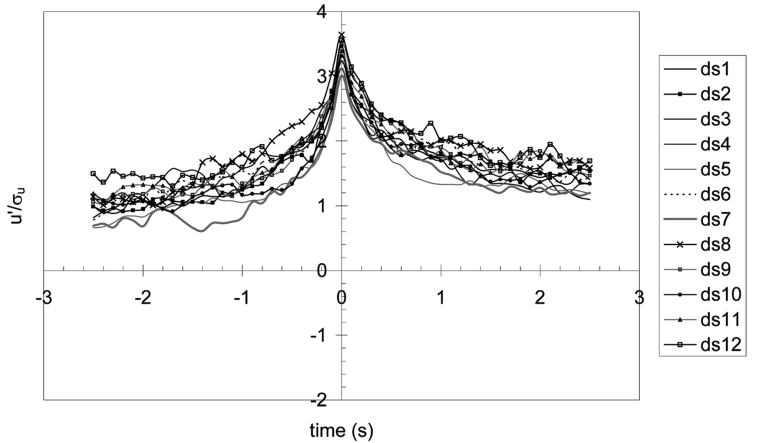

(a) Streamwise velocity component

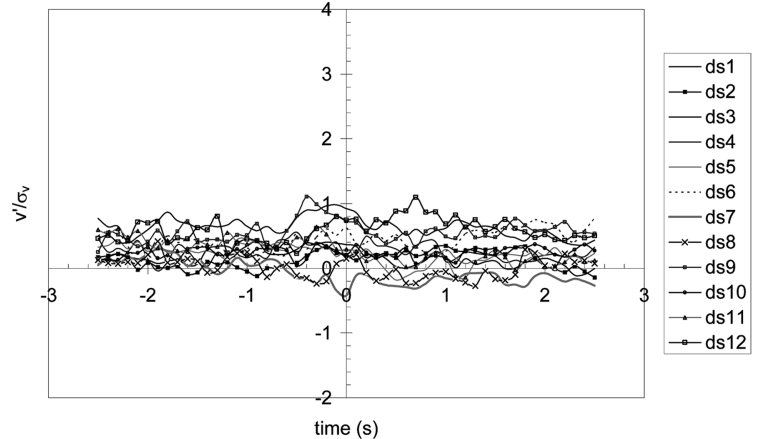

(b) Lateral velocity component

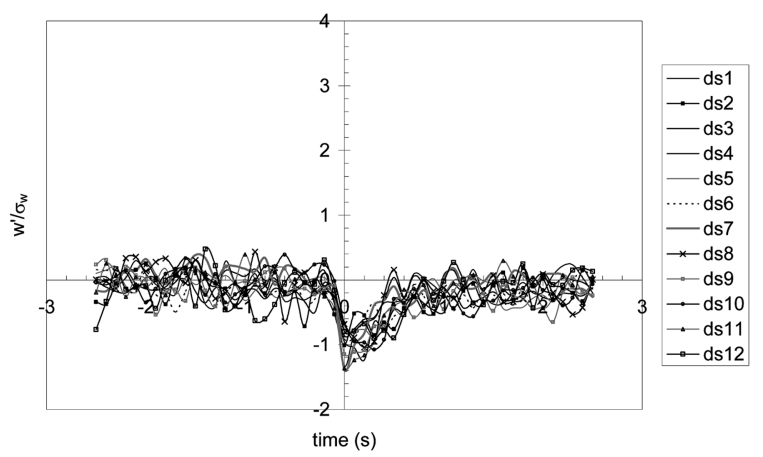

(c) Vertical velocity component

Fig. 1 Conditional sampling of the peak velocity components using an anemometer $1 \mathrm{~m}$ above the ground corresponding to times when the velocity was greater than $99.95^{\text {th }}$ percentile value of the streamwise component (Sterling, et al. 2006).

motion, or fourth quadrant fluctuation (Willmarth and $\mathrm{Lu} \mathrm{1972).}$

Figs. 2-5 illustrate the time evolving $u^{\prime}$ and $w^{\prime}$ vector diagrams corresponding to an ensemble average peak event detected by the ultrasonic anemometers at $1 \mathrm{~m}, 3 \mathrm{~m}, 6 \mathrm{~m}$ and $10 \mathrm{~m}$ above the ground respectively. In Figs. 2-5 the time scale has been altered to a spatial scale. In order to get an insight into the flow structure from single point velocity measurements, Taylor's hypothesis of "frozen flow" was adopted. This hypothesis implies the need of a uniform mean convection velocity. Hence the mean velocity obtained from the anemometer at $10 \mathrm{~m}$ above the ground was subtracted from all of the other anemometer datasets. As can be seen from Table 1 this was the order of $10 \mathrm{~m} / \mathrm{s}$, which is the assumed convection velocity. The four time series in each of the figures correspond to the data obtained from the four anemometers. The time evolution of longitudinal and vertical velocity fluctuations, shown in Figs. 1(a) and 1(c), corresponds to the velocity vectors at $z=1 \mathrm{~m}$ in Fig. 2. All figures clearly highlight the presence of a sweeping or downburst event close to $t=0$ and support the results of Fig. 1 . The events detected by anemometers 6 and 10 (Figs. 4 and 5) also illustrate how the sweeping motion can induce additional rotation within the flow field at lower heights above the ground. It is hypothesized that these structures are also present in a smaller scale and as a result of the experimental setup are not so evident in Figs. 2 and 3. The combined effect of the sweeping motion and increased local 


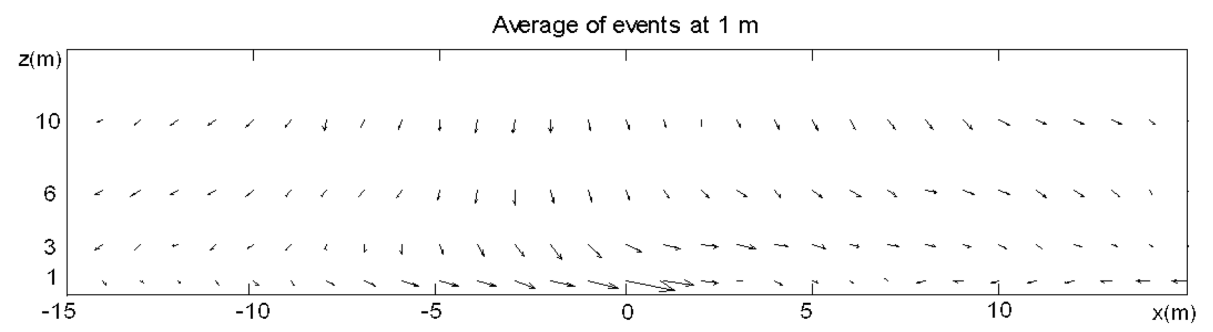

Fig. 2 Spatial structure of the velocity minus the event mean velocity for an event detected $1 \mathrm{~m}$ above the ground

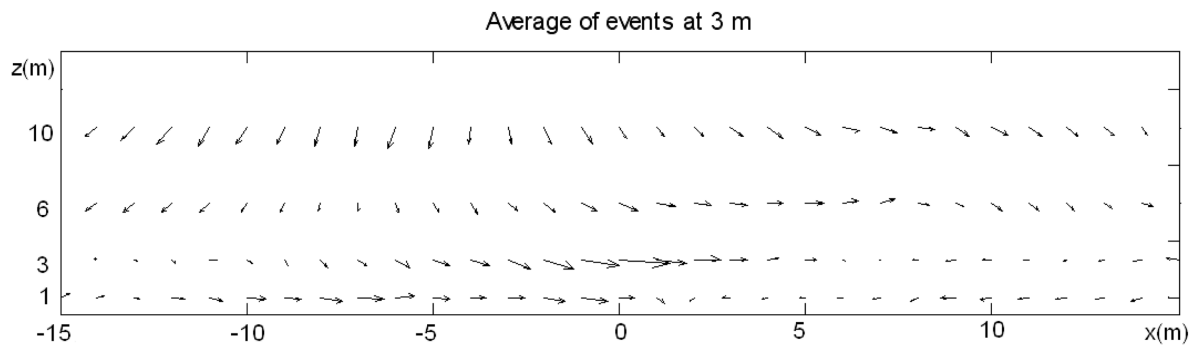

Fig. 3 Spatial structure of the velocity minus the event mean velocity for an event detected $3 \mathrm{~m}$ above the ground

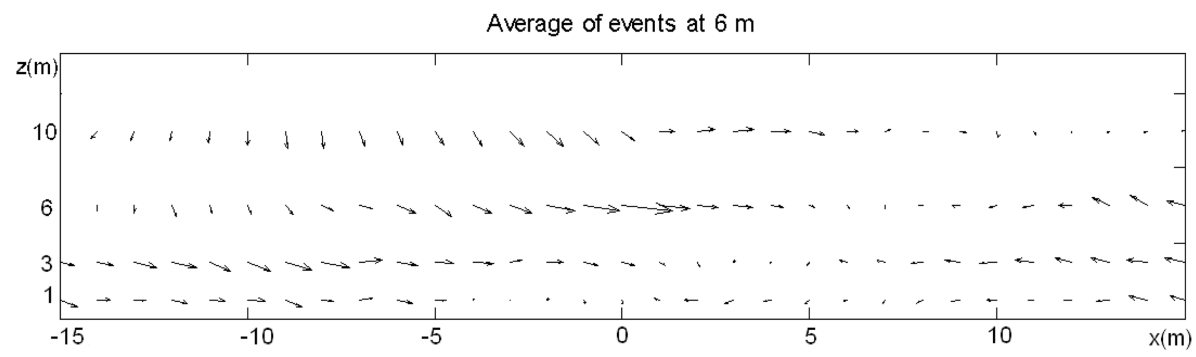

Fig. 4 Spatial structure of the velocity minus the event mean velocity for an event detected $6 \mathrm{~m}$ above the ground

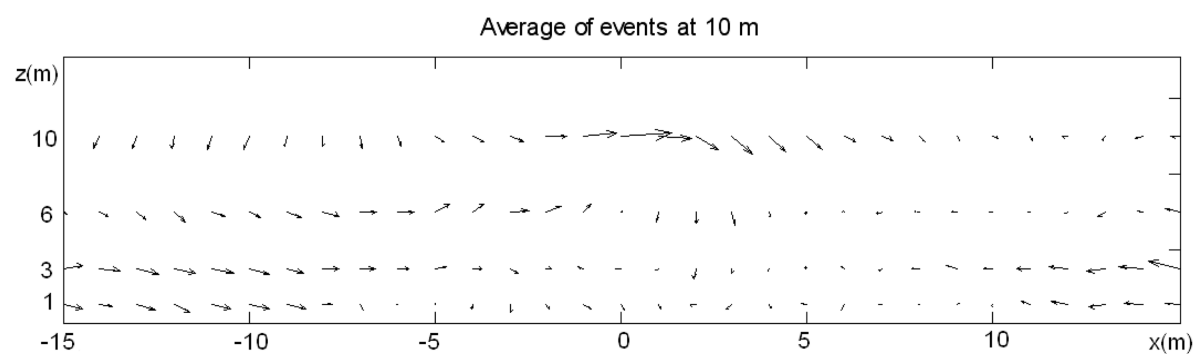

Fig. 5 Spatial structure of the velocity minus the event mean velocity for an event detected $10 \mathrm{~m}$ above the ground

rotation will produce an increase in the horizontal velocity peak.

To a large extent the analysis presented above is not new (with the exception of Figs. 2-5) and has been provided for the benefit of the reader. In the above it has been assumed that although there is 
variability between individual events, the underlying, average behaviour is consistent. Typically, this is not an unreasonable assumption to make since the inherent turbulence of the flow requires some sort of averaging to be undertaken. However, in the following section this ensemble averaging of all of the events is examined and found in this instance to be a rather questionable assumption. For example, the apparent circulation illustrated clearly in Fig. 5 around the event time is not evident in all of the events.

\section{Ensemble averaging and flow classification}

Fig. 1(a) illustrates the asymmetry of the evolution of the ensemble averaged gust profile of the normalised streamwise velocity component. In order to examine if this asymmetry occurs in all of the individual events, a symmetry parameter, $S$ defined in Eq. (1) has been developed.

$$
S=\frac{\frac{1}{T}\left[\int_{0}^{T / 2} u(t) d t-\int_{-T / 2}^{0} u(t) d t\right]}{\sigma_{e v}}
$$

where $T$ represents the length of the mini time series of each identified event, $\sigma_{e v}$ is the standard deviation of each event, and the remaining symbols take their usual meaning. If the time series in question is symmetrical, then the corresponding value of $S$ would be zero. If the time series corresponds to a ramp-step function as illustrated in Fig. 6, the corresponding value of $S$ is $\sqrt{(3 / 5)} \approx 0.775$. In order to classify the events in the current analysis it was decided that a value of $S=|0.39|$, i.e., half that of a ramp-step function, would be adopted. Hence, if $S<-0.39$ the event represented a backward facing ramp-step and will be termed a type one event; if $-0.39<S<0.39$ the events were considered to be symmetrical, classified as a type two event; and if $S>0.39$ the event was considered to correspond to a (forward facing) ramp-step function, a type three event.

Fig. 7 illustrates the normalised streamwise and vertical velocity components of three individual type I, type II and type III events as identified from an inspection of the traces of the individual events. The different behaviour in the evolution of the horizontal velocity component is clearly evident.

The results of this analysis for all of the data are given in Table 2. It is interesting to note that using the above classification method, the majority of the events appear to be symmetrical, i.e., more than $50 \%$ of the events are classified as type II, followed by events of type III, which appear twice more often than those of type I. Thus when ensemble averaged type II events are weighted more heavily than either type I or type III and lead to the asymmetry reported in Sterling, et al.

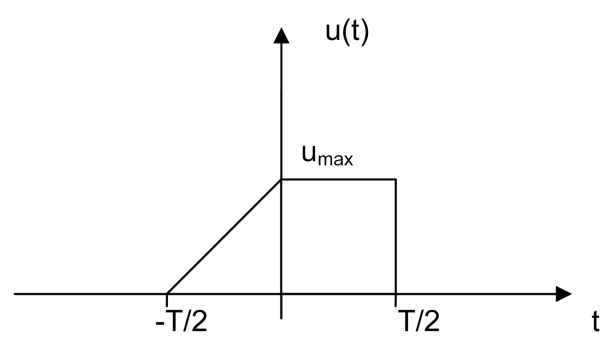

Fig. 6 A ramp-step function illustrating a type 3 event. $u_{\max }$ represents the maximum velocity of the local time series 

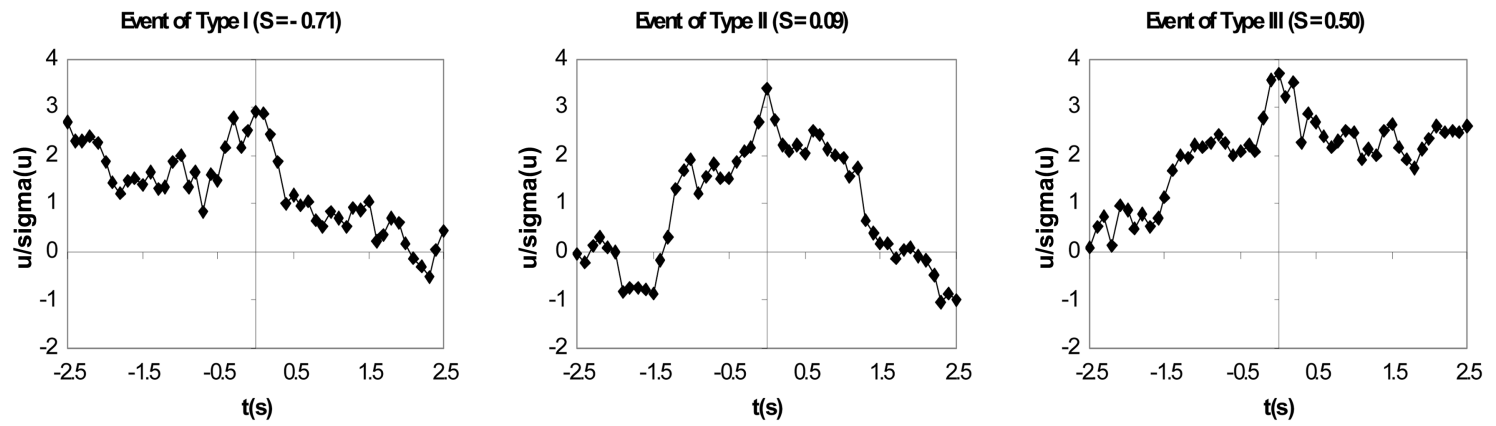

Fig. 7 Velocity distributions of individual flow events detected at $z=6 \mathrm{~m}$, illustrating the three different event types (Data obtained from $d s 1$ using the anemometer at $z=6 \mathrm{~m}$ as a trigger)

Table 2 Number (percentage) of each type of event at different heights above the ground

\begin{tabular}{ccccc}
\hline $\begin{array}{c}\text { Height above } \\
\text { ground }(\mathrm{m})\end{array}$ & $\begin{array}{c}\text { Type I } \\
(S<-0.39)\end{array}$ & $\begin{array}{c}\text { Type II } \\
(-0.39<S<0.39)\end{array}$ & $\begin{array}{c}\text { Type III } \\
(S>0.39)\end{array}$ & Total \\
\hline 1 & $38(9.0 \%)$ & $258(60.5 \%)$ & $130(30.5 \%)$ & $426(100 \%)$ \\
3 & $46(13.6 \%)$ & $185(54.7 \%)$ & $107(31.6 \%)$ & $338(100 \%)$ \\
6 & $58(17.0 \%)$ & $174(51.0 \%)$ & $109(32.0 \%)$ & $341(100 \%)$ \\
10 & $67(20.7 \%)$ & $170(52.6 \%)$ & $86(26.6 \%)$ & $323(100 \%)$ \\
\hline
\end{tabular}

(2006). Table 2 also illustrates that the frequency the Type I events appear to increase with height, while the type II events decrease. It is interesting to note that Fig. 7 illustrates that the peak in streamwise velocity occurs over a relatively short time period and as such confirms that concept of the high frequency events superimposed on a low frequency event is not a consequence of the ensemble averaging procedure but of a physical event.

Fig. 8 illustrates the ensemble average of all of the mini time series associated with an event group by type. The results are similar to those of Fig. 7. It can be seen that except for the gusts measured at $1 \mathrm{~m}$, for which the normalised velocity increase and decrease is sharper, the time evolution of the normalised horizontal velocity is relatively independent of height, and each type of gust can be well fitted for all heights by unique analytical expressions. It is interesting to note that all of the events are associated with a rapid change in the vertical velocity component at the event time.

Fig. 9 shows in more detail the number of events at all heights for different values of $S$. The mean value of $S$ for all events was found to be 0.2 , but the highest probability is for ramp-step like behaviour of $u$, with $S$ approximately 0.6 . It is evident from Fig. 9 that asymmetry of the events follows a continuum, i.e., the division of the behaviour into three event types is somewhat arbitrary but is adopted for the purposes of this analysis.

Fig. 10 illustrates the spatial structure of the ensemble average of events of type I, II and III respectively, detected at $z=6 \mathrm{~m}$, with the mean velocity of the events subtracted in order to aid with visualisation. As shown in Fig. 8 it is evident that following all of the events there is a significant downward component of velocity. For type I events, for $t<-0.5 \mathrm{~s}$ the flow appears to be diverted upwards in front of the downburst and before the maximum velocity is reached, generating a 

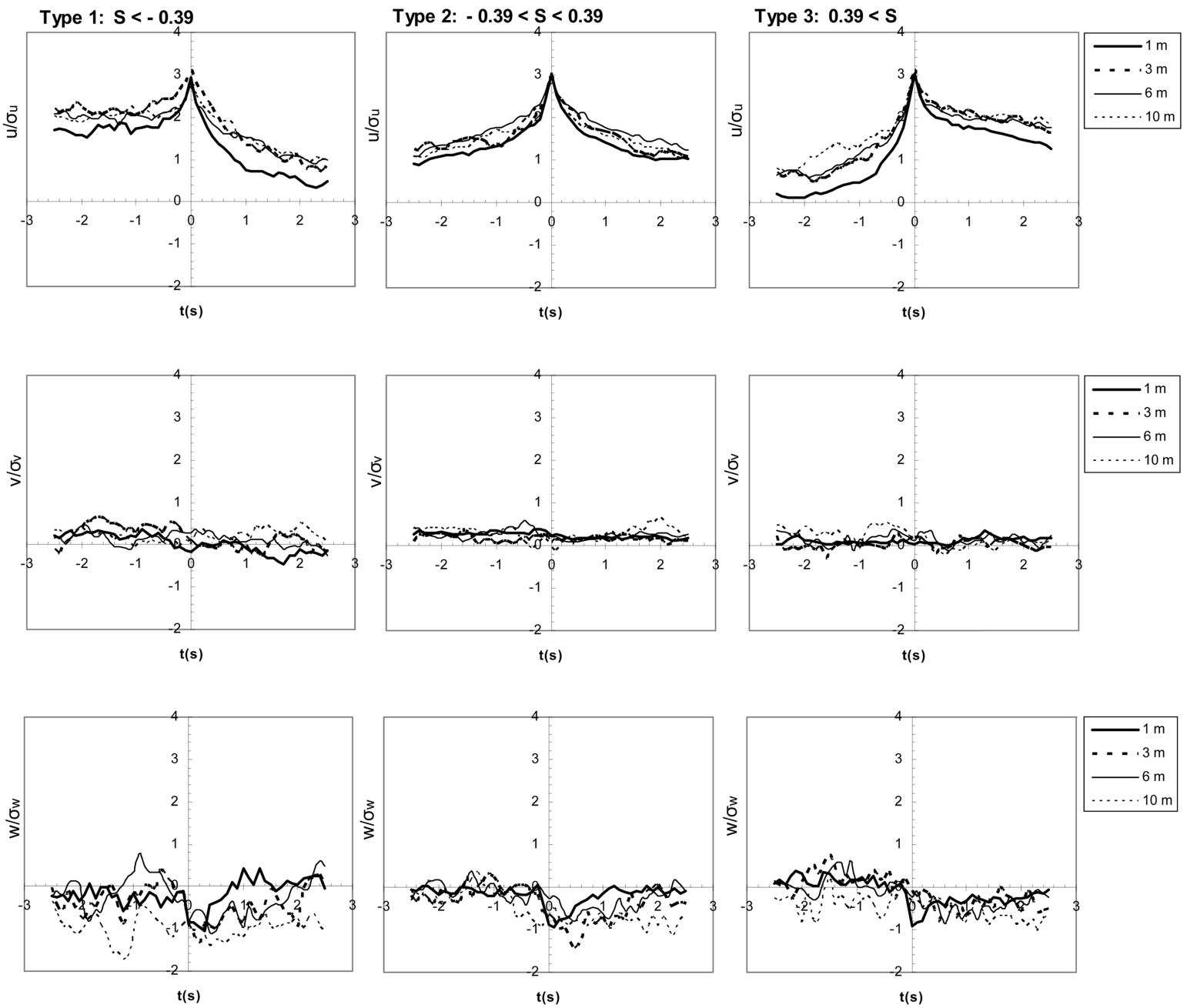

Fig. 8 Ensemble averages of events of type I, II and III respectively

clockwise circulation pattern that resembles that of horseshoe vortices frequently detected in boundary layer flows (McNaughton 2004, Skote, et al. 2002), while the downward flow following the $u$ peak is present in all event types.

In the more frequent events of type II and III, this vortex is not noticeable, and instead a region of convergence (negative value of $d u / d x+d w / d z$ ) appears preceding the downward flow. Convergence in the $x-z$ plane implies some lateral outflow, which, due to the symmetry, is on average zero. For type III events, a clear convergence front with an inclination of approximately 56 degrees can be identified, showing the boundary of the incoming faster mass of air. This inclined front causes a stagger in the velocity maxima at the different heights, as illustrated in Fig. 11. For events of type III detected at $6 \mathrm{~m}$, the maximum in $u$ at $3 \mathrm{~m}$ appears 1 second after the trigger peak at $6 \mathrm{~m}$, and the maximum at $1 \mathrm{~m}, 1.5$ seconds after the trigger peak.

If it is assumed that the measured events resemble a hairpin or horseshoe type of structure then it is possible that the different types of events represent slices through different parts of the structure, 


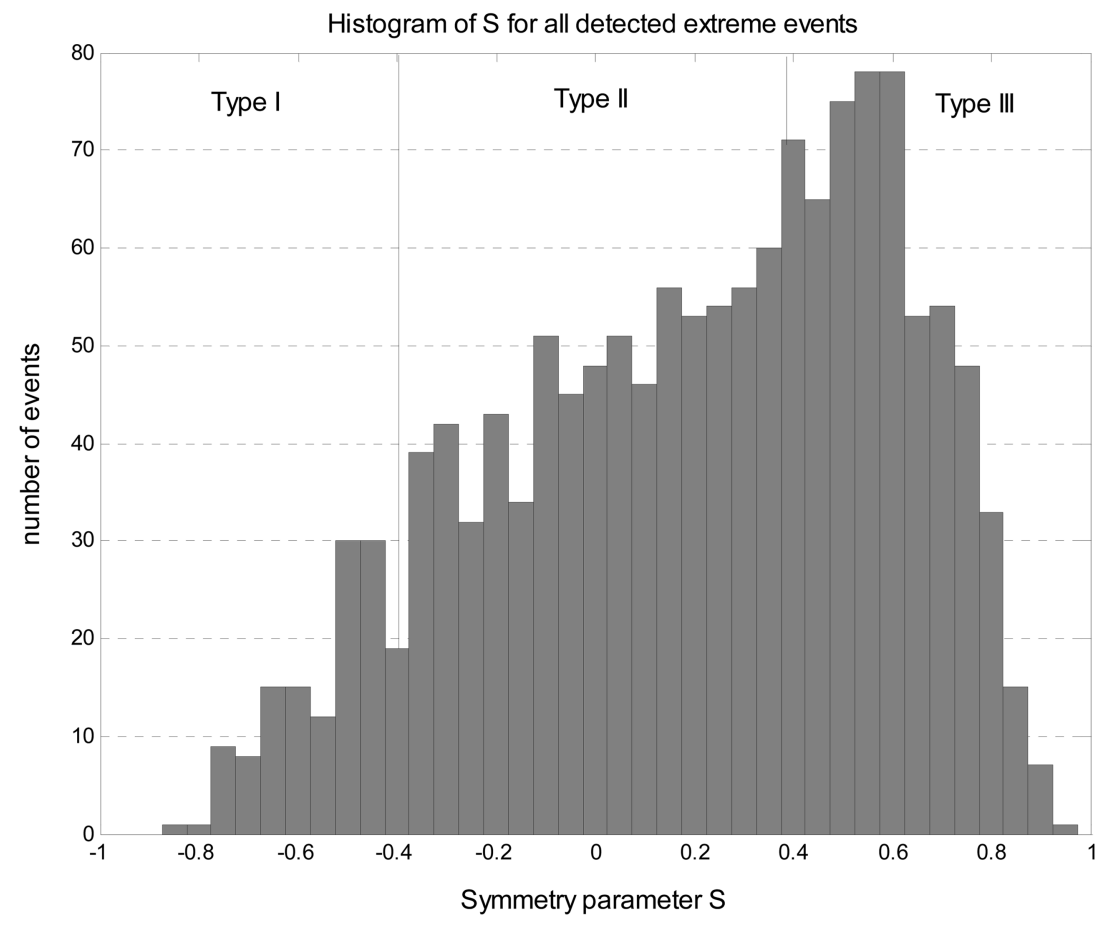

Fig. 9 Number of events per value of symmetry parameter for all heights
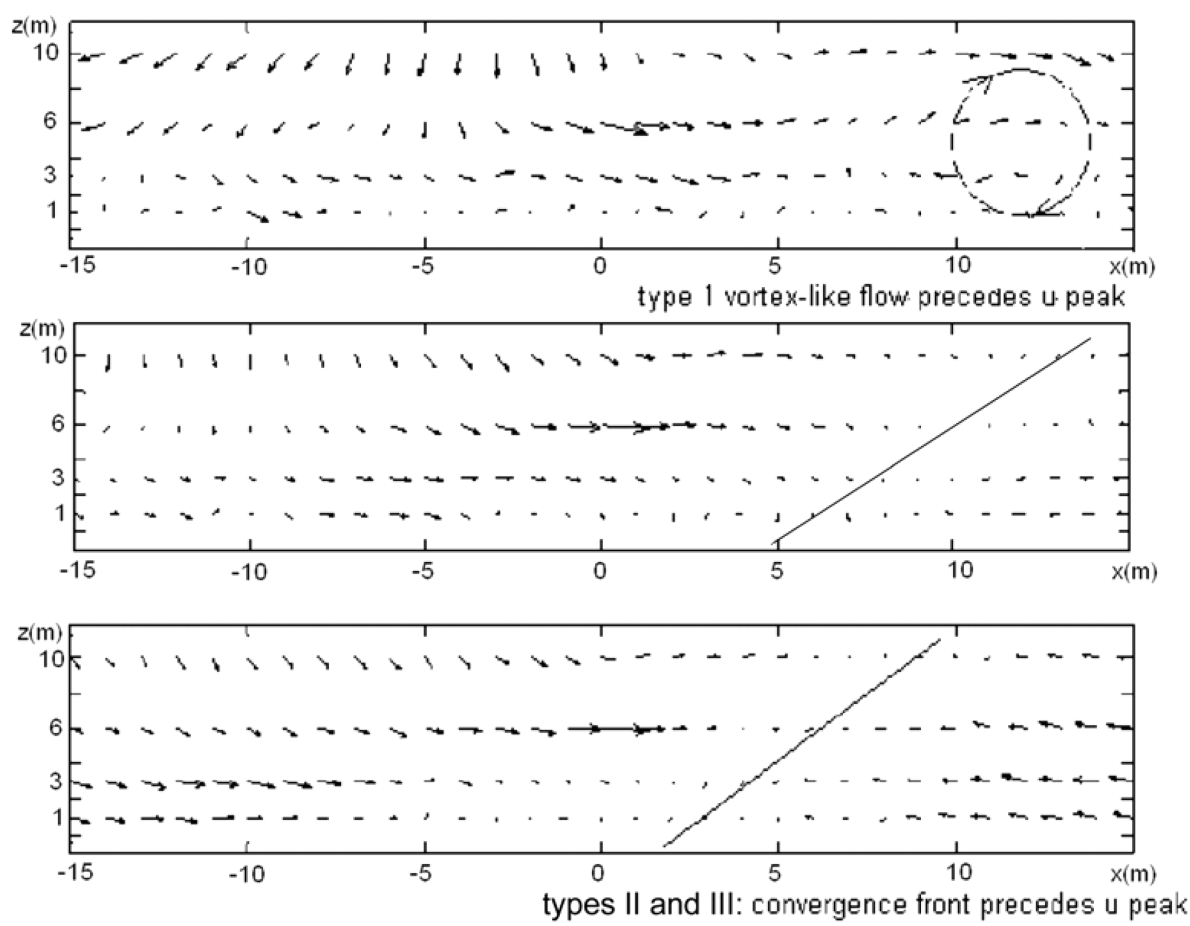

Fig. 10 Spatial structure of events of type I, II and III, detected at $6 \mathrm{~m}$ 

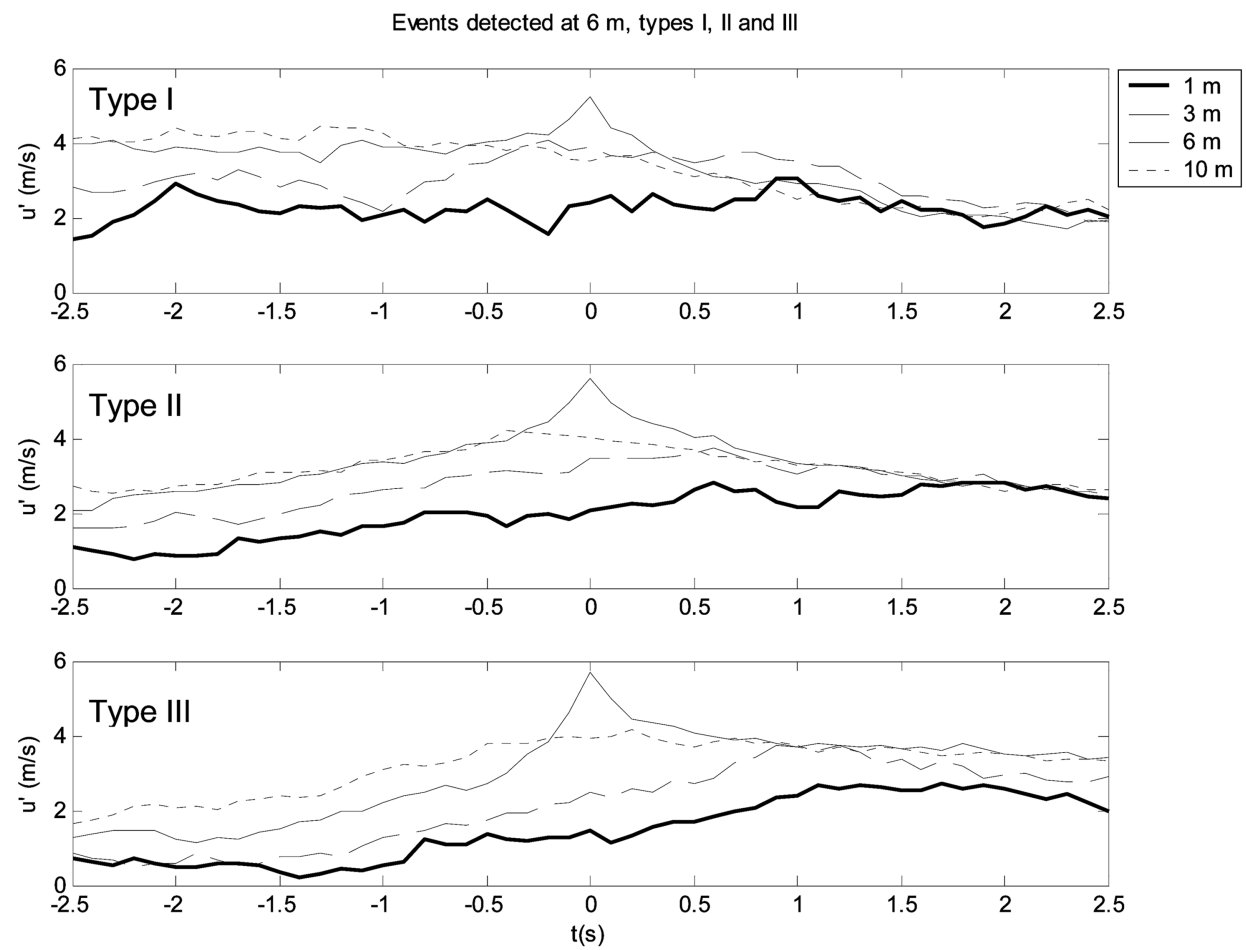

Fig. 11 Ensemble average streamwise velocity distributions per event type for a trigger at $6 \mathrm{~m}$

e.g. the head or legs. This would help to explain the observed differences and the results of Fig. 9.

The above section has clearly illustrated that at the extreme the flow structures exhibit different types of behaviour. The following section will examine if any simple model can be used to explain the main features identified.

\section{Modelling}

It is often assumed that the flow within the atmospheric boundary layer is dominated by hairpin vortex structures. Downburst structures have recently received renewed interested in the wind engineering community and recourse to an empirical model of such a structure is made in order to examine if such flow phenomena can explain any of the flow behaviour observed above. In addition, an idealised hairpin structure has been developed in order to examine if these types of models can explain any of the behaviour illustrated above.

\subsection{An empirical downburst model}

Fig. 10 indicates that the flow possess a strong downwards component and as such it is perhaps not unreasonable to investigate if a downburst model is capable of predicting the observed behaviour. Oseguera and Bowles (1988) developed an empirical model of the streamwise and vertical velocity components of a downburst. The four parameters that define the downburst geometry and intensity are: 1) a characteristic horizontal dimension; 2) a maximum wind velocity; 
3) an altitude of maximum outflow; and 4) a depth of outflow, as illustrated in Eq. (3).

$$
\begin{aligned}
& u=\frac{\lambda R^{2}}{2 r}\left[1-e^{-(r / R)^{2}}\right]\left(e^{-z / z^{*}}-e^{-z / \varepsilon}\right) \\
& w=-\lambda e^{-(r / R)^{2}}\left[\varepsilon\left(e^{-z / \varepsilon}-1\right)-z\left(e^{-z / z^{*}}-1\right)\right]
\end{aligned}
$$

where $r$ is the radial coordinate, (distance from downburst centre), $z$ is the height above the ground, $u$ is the horizontal velocity in radial direction, $w$ is the vertical velocity, $\lambda$ is a scaling factor, $\varepsilon$ is a characteristic height in the boundary layer, $z^{*}$ is another characteristic height, the altitude at which the horizontal velocity increase is half the maximum value, and $R$ is the radius of downburst shaft. If this downburst is convected by the flow assuming "frozen" turbulence, it will produce the flow pattern and velocity fluctuations shown in Fig. 12.

Compared with the normalised velocities shown in Fig. 8, it can be observed that the downburst introduces negative values of $w$ after the maximum in $u$, a characteristic shared by all the studied types of extreme events. The slow increase and more rapid decrease in $u$ resembles events of type I. However, this model does not reproduce the sharp peaks characteristic of most extreme events.

It is acknowledged that there are other downburst models available which may provide sharper peak characteristics to those examined above. However, the horseshoe vortex is often considered as a possible mechanism capable of describing vortex motion. Also, the work of Oseguera and Bowles
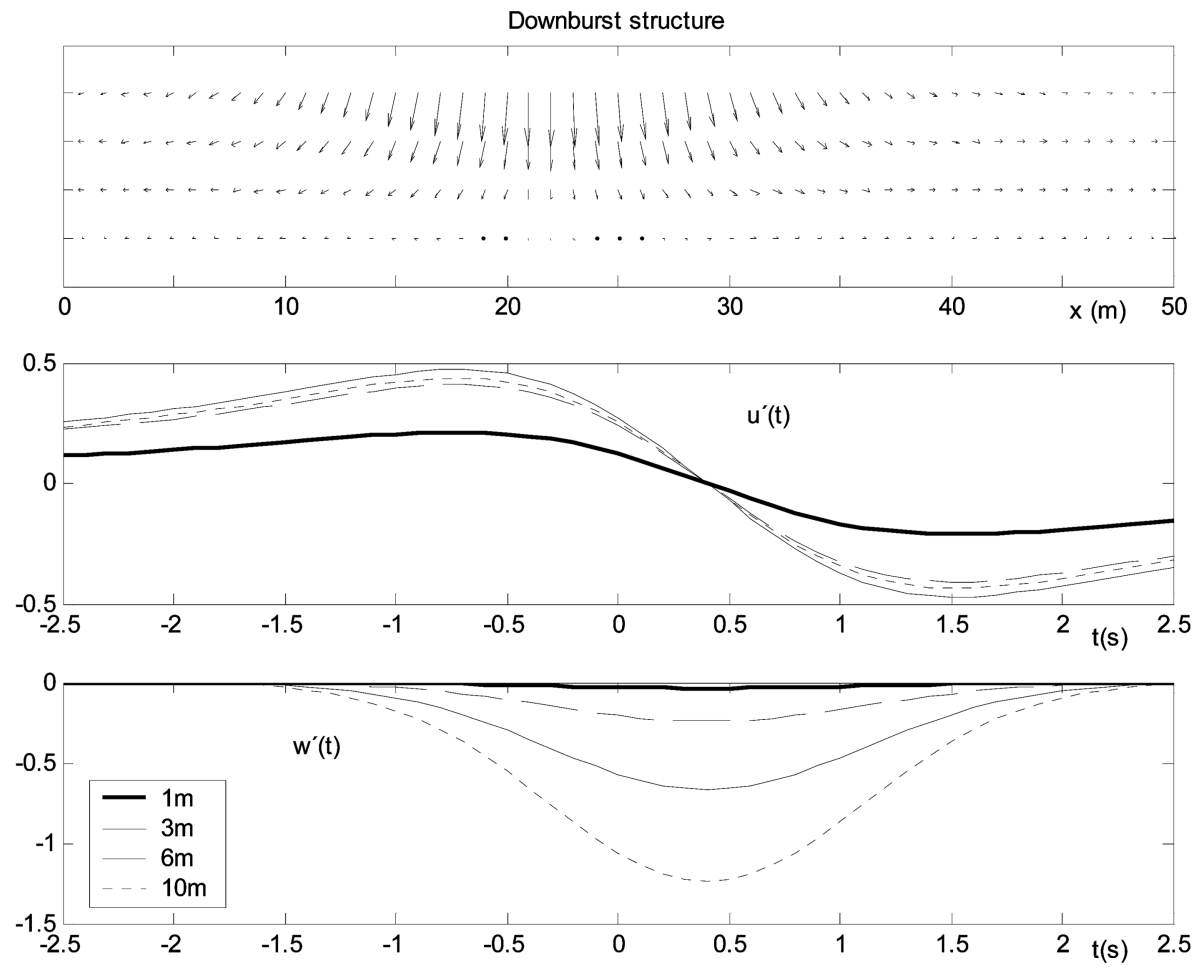

Fig. 12 Results of Eq. (3): Spatial structure of an ideal downburst and longitudinal and vertical velocities induced if the structure were convected under Taylor's hypothesis of "frozen turbulence" 


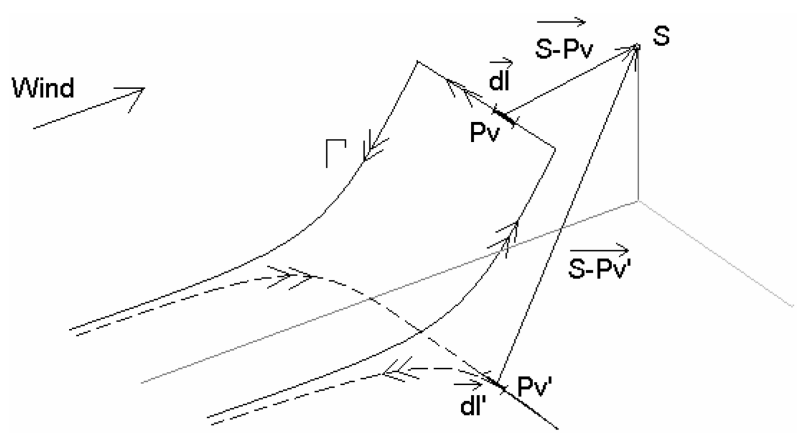

Fig. 13 An idealised model of a hairpin vortex structure

(1988) is frequently cited as a possible downburst model (e.g. Chay, et al. 2006), perhaps due to the relatively simple equations for the vertical and horizontal components of velocity. It is envisaged that additional models will be the subject of future work

\subsection{An idealised horseshoe structure}

Horseshoe, or hairpin vortices have been consistently detected in boundary layers and channel flows, either in numerical simulations (Ovenden and Smith 2003, Zhou, et al. 1999) or in physical experiments (Adrian, et al. 2000). McNaughton (2004) proposed such structures as a fundamental element of wall bounded shear turbulence. Hence, it seems appropriate to explore the possibility that the detected extreme events could be produced by the passage of a vortex of this kind.

An ideal horseshoe vortex was modelled with the shape as in Fig. 13: a straight segment as the vortex "head", and two curved "legs", shaped as an exponential functions but of finite length. A viscous core of finite radius prevents the velocity for becoming infinity. An image vortex simulates the effect of the floor. The vortex is convected by the stream with the mean velocity of the flow.

At an instant $t$, a line element of length $d l$ centred in point $P v$ of the vortex line of strength $\Gamma$ induces in point $S$ a velocity $\mathbf{d V}$, that can be computed by the Biot-Savart law:

$$
\overrightarrow{\mathbf{d V}}=\frac{\Gamma}{4 \pi} \frac{\overrightarrow{d l} \times(\overrightarrow{S-P v})}{|S-P v|^{3}}
$$

In the same way, a line element in point $P v^{\prime}$ of the mirrored vortex contributes to the induced velocity with

$$
\overrightarrow{\mathbf{d} \mathbf{V}^{\prime}}=\frac{\Gamma}{4 \pi} \frac{\overrightarrow{d l^{\prime}} \times\left(\overrightarrow{S-P v^{\prime}}\right)}{\left|S-P v^{\prime}\right|^{3}}
$$

Adding the contribution of all the vortex and mirrored vortex line elements, the induced velocity vector $\mathbf{V}$ in instant $t$ is obtained. The process is repeated for each instant $t$, while the vortex is convected by the mean velocity. Fig. 14 shows the flow pattern and the velocity components that would be recorded by four anemometers at $1,3,6$ and $10 \mathrm{~m}$ in two different positions $(y=4 \mathrm{~m}$, between the vortex legs and $y=6 \mathrm{~m}$, outside) with respect to a theoretical horseshoe vortex with its head core $5.2 \mathrm{~m}$ above the ground, its legs at $y=-5$ and $y=5 \mathrm{~m}$, a viscous radius of $1 \mathrm{~m}$ and the 

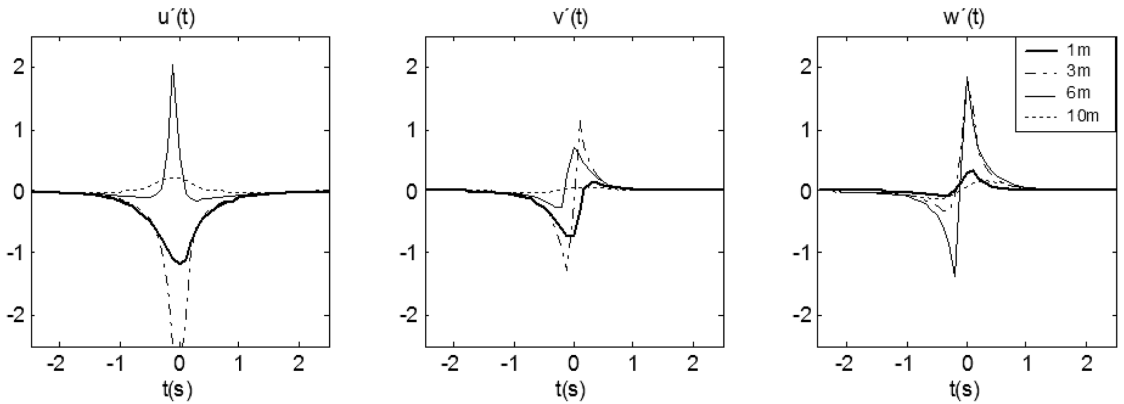

(a) Anemometers located between the vortex legs, at $y=4 \mathrm{~m}, \mathrm{z}=1,3,6$ and $10 \mathrm{~m}$
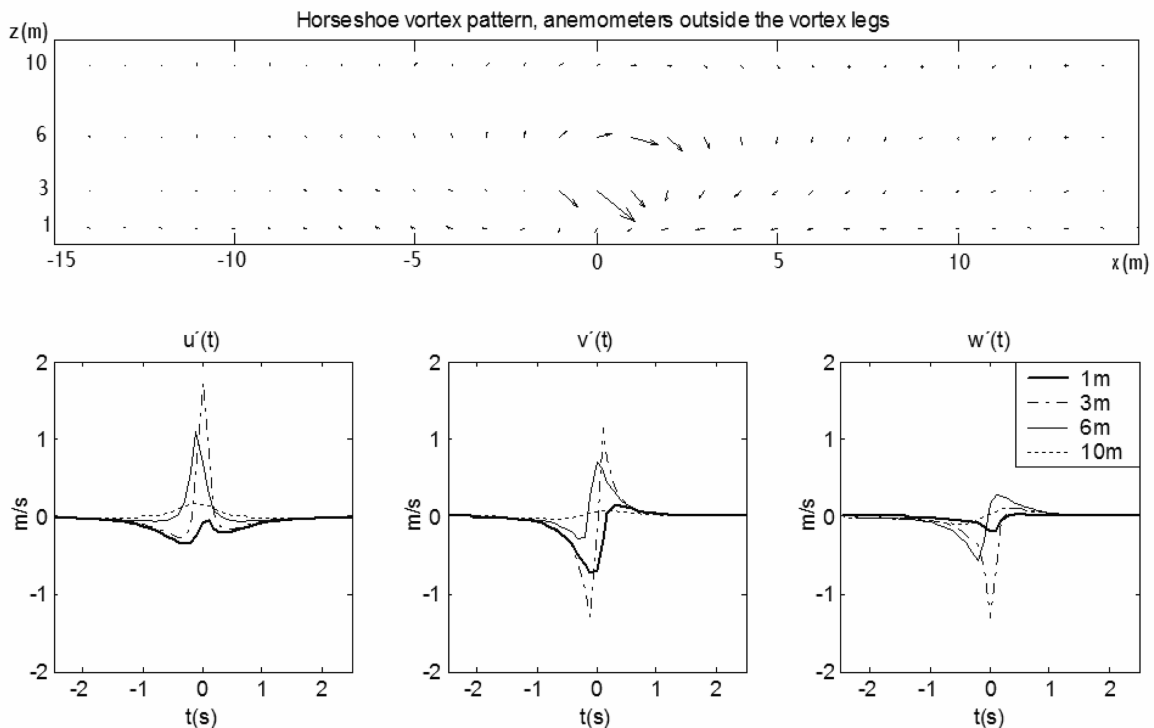

(b) Anemometers located outside of the vortex legs, at $y=6 m, z=1,3,6$ and $10 \mathrm{~m}$

Fig. 14 Streamwise velocity distributions obtained when an idealised horseshoe vortex is convected past the anemometers

circulation, $\Gamma=20 \mathrm{~m}^{2} \mathrm{~s}^{-1}$.

Fig. 14 shows that the longitudinal velocity above the vortex head $(6 \mathrm{~m})$ increases as expected with a sharp peak, and a closer inspection also reveals a slight asymmetry like in type III events. However, the behaviour of the vertical velocity is opposite to what was found in the studied extreme events. The upward flow between the legs that follows the hairpin head could perhaps be masked by the stronger downward flow that could originate these vortices, but this is pure speculation. The negative longitudinal velocity induced by these vortices below the head does not match the data either.

If the structure passes on one side of the anemometers, there is little upward flow, since the legs also induce negative vertical velocities in this region. The main differences with the induced flow and the detected events is that the negative vertical velocity reaches a sharp peak simultaneously with the positive peak in $u$, instead of a smoother minimum with some delay found in the field 

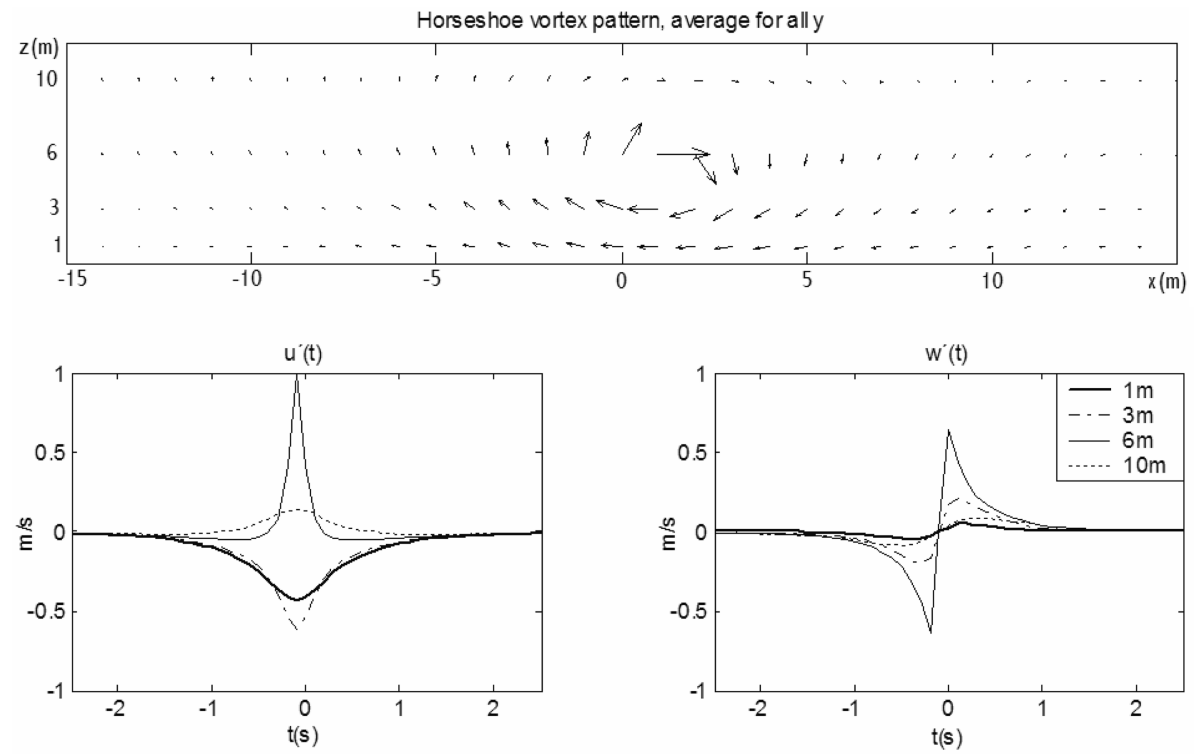

Fig. 15 Streamwise $y$-averaged velocity distributions obtained when an idealised horseshoe vortex is convected past the anemometers

measurements.

If the velocity fields for all possible distances $y$ from the horseshoe vortex centre are averaged, the lateral velocity vanishes because of symmetry, and the averaged flow configuration is as shown in Fig. 15. This picture resembles Fig. 13, but the evolution of $u(t)$ is now completely symmetrical around its peak and the evolution of $w$ is completely antisymmetrical, since the influence of the legs is cancelled by the averaging procedure. There is also no time lag between the maxima or minima at different heights, as it is the case for measurements in a single $y$ position, because this lag is also introduced by the lateral vortex legs. The upward flow induced behind the head by the legs is also cancelled in the average by the downward flow induced outside the legs, leaving only the velocity field induced by the vortex head. The similarity with Fig. 13 comes from the strong influence of the vortex head between the legs.

Hence, it is possible to reproduce some features of the observed flow by assuming an idealised horseshoe structure. However, the localised increase in the streamwise velocity which in reality occurs across all of the measured heights is not reproduced. This coupled with the modelled behaviour of the vertical velocity represents a major drawback with this type of simplified model.

\subsection{Empirical modelling}

Fig. 8 illustrates that the ensemble average behaviour of the velocity components sorted by type follow similar trends across all heights with relatively little scatter. Attempts to model this behaviour using an idealised horseshoe vortex and an empirical downburst model have been undertaking with limited success. Hence, an empirical model, essentially a curve fit to the data, is developed below for each of the three types of events, in the hope that such an approach might ultimately be useful in terms of codification and design. 
In keeping with the approach adopted by Sterling, et al. (2006) the experimental curves of Fig. 8 are fitted with simple analytical expressions that can be employed either to model a single gust of any of the three types proposed, or can be inserted in stochastic wind simulations, considering the different probability of each type. Attention is restricted to the streamwise and velocity components since no significant behaviour for the lateral vertical velocity component was observed. As illustrated in Fig. 16, expressions of the form:

$$
\frac{u^{\prime}}{\sigma_{u}}(t)=A \cdot e^{-\left(\frac{|t| t}{B}\right)^{k}} \quad \frac{w^{\prime}}{\sigma_{w}}(t)=M \cdot e^{-\left(\frac{t-D}{C}\right)^{2}}
$$

were fitted to the data. In Eq. (6) the parameters $A, B, C, D$ and $k$ represent constants and the remaining symbols are as previously defined. Appropriate values for these parameters are shown in Table 3.
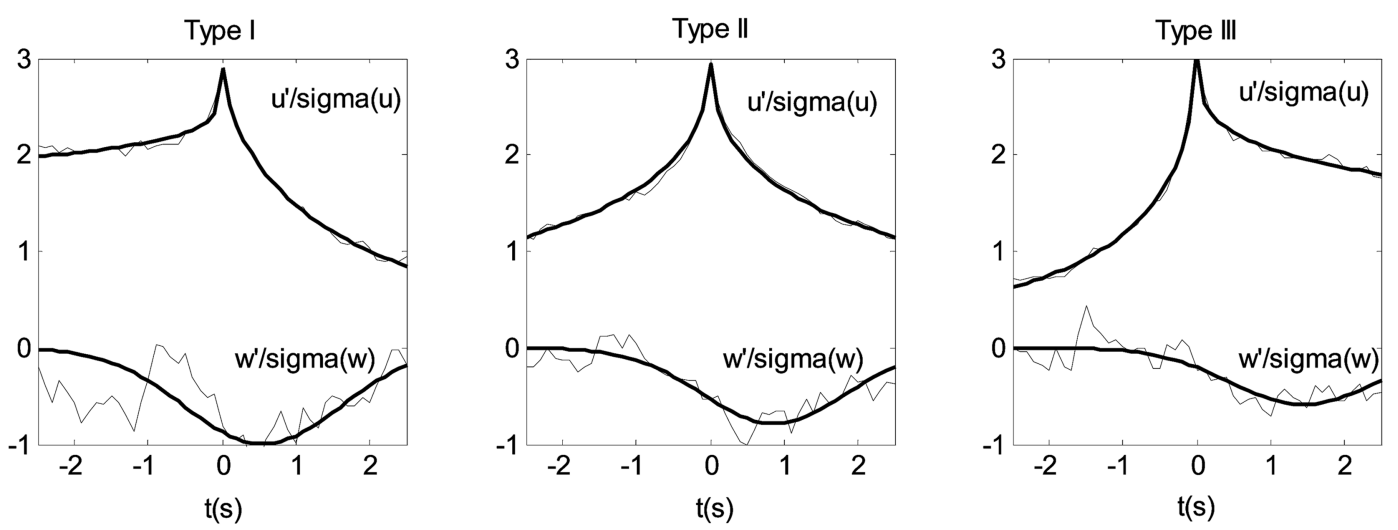

Fig. 16 Average of normalised events at heights 3,6 and $10 \mathrm{~m}$ sorted by type, and analytical fitting. The vertical scale represents the value of normalised velocity

Table 3 Constants corresponding to Eq. (6)

\begin{tabular}{|c|c|c|c|}
\hline & \multicolumn{2}{|c|}{ Streamwise } & \multirow{2}{*}{ Vertical } \\
\hline & $t<0$ & $t>0$ & \\
\hline \multirow{3}{*}{ Type I } & $\mathrm{A}=2.90$ & $\mathrm{~A}=2.90$ & $M=-1.0$ \\
\hline & $B=160$ & $\mathrm{~B}=1.82$ & $\mathrm{D}=0.55$ \\
\hline & $\mathrm{K}=0.23$ & $K=0.66$ & $C=1.50$ \\
\hline \multirow{3}{*}{ Type II } & $\mathrm{A}=2.94$ & $A=2.94$ & $\mathrm{M}=-0.78$ \\
\hline & $\mathrm{B}=2.83$ & $\mathrm{~B}=2.83$ & $\mathrm{D}=0.87$ \\
\hline & $\mathrm{K}=0.52$ & $K=0.52$ & $\mathrm{C}=1.39$ \\
\hline \multirow{3}{*}{ Type III } & $\mathrm{A}=3.04$ & $\mathrm{~A}=3.04$ & $\mathrm{M}=-0.58$ \\
\hline & $\mathrm{B}=1.08$ & $\mathrm{~B}=17.72$ & $\mathrm{D}=1.46$ \\
\hline & $\mathrm{K}=0.55$ & $\mathrm{~K}=0.33$ & $\mathrm{C}=1.42$ \\
\hline
\end{tabular}




\section{Conclusions}

Previously published experimental data has been reanalysed using a newly defined symmetry parameter. The results of this analysis have indicated that during extreme events, three different types of flow behaviour occur (although it is acknowledged that the division of the events into three different types is somewhat arbitrary). The distribution in localised streamwise velocity has been shown to correspond to either a forward facing ramp-step function, a backward facing ramp-step function or a symmetrical profile. The distribution of these events is skewed towards a forward facing (type III) ramp structure as illustrated in Fig. 9 and Table 2. In all cases it is interesting to note that the behaviour of the vertical velocity is similar, i.e., there is significant down-flow corresponding to the time of the event. Velocity vector diagrams similar to those of Fig. 10 confirm this flow classification captures different physical events and the observed behaviour is not due to the conditional sampling technique.

Using Taylor's frozen turbulence hypothesis the spatial structure of the three types of events has been observed (see Fig. 10). For type I events the flow appears to be directed upwards prior to the event which generates a clockwise circulation pattern. In the more frequent events of type II and III, this circulation is not noticeable, and instead a region of convergence (negative value of $d u / d x+d w /$ $d z$ ) appears preceding the downward flow. Convergence in the $x-z$ plane implies some lateral outflow, which, due to the symmetry, is on average zero. For type II and III events, a clear convergence front with an inclination between 45 and 65 degrees can be identified, showing the boundary of the incoming faster mass of air.

With most types of boundary layer flows, horseshoe vortex structures have been shown to occur frequently and it is not unreasonable to initially assume that the extreme events may occur as a result of such structures. In order to investigate this, an idealised horseshoe vortex was modelled with the shape as shown in Fig. 13: the vortex was assumed to be convected by the stream with constant velocity. Figs. 14(a) and 14(b) clearly illustrate the difference in velocity distributions obtained with respect to the location of the observer. However, this simple model was unable to correctly predict the vertical velocity component and illustrated a behaviour opposite to that recorded in reality. An empirical model of a downburst has also been investigated. Although this model produced the correct behaviour in vertical velocity component, the streamwise velocity was opposite to that measured.

The results of Table 2 illustrate that a realistic gust model should consider that at least $30 \%$ of the wind peaks that exceed the $99.95 \%$ confidence value, do not decay rapidly, but are followed by an increase of 2-3 $\sigma_{u}$ above the mean velocity, with the opposite behaviour for $10-15 \%$ of the gusts. An empirical model of the flow behaviour has been developed which enables a single gust of a particular type to be modelled. Although this model has no underlying theory it provides a simple way of reproducing the correct flow behaviour and could be used in isolation or perhaps as part of a stochastic wind simulation.

\section{Acknowledgements}

Ana Scarabino would like to thank Mr. Federico Bacchi, who ran numerical simulations of downward flows, helping to highlight some features of the detected gusts. She is also most grateful to Dr. Mark Sterling and Prof. Chris Baker for their kind invitation from the University of Birmingham, that made it possible for her to participate in this work. 


\section{References}

Adrian, R. J., Meinhart, C.D. and Tomkins, C. D. (2000), "Vortex organization in the outer region of the turbulent boundary layer", J. Fluid Mech., 422, 1-54.

Baker, C. J. (2001), "Unsteady wind loading on a wall”, Wind and Struct., 4(5), 413-440.

Chay, M. T., Albermania, F. and Wilson, R. (2006), "Numerical and analytical simulation of downburst wind loads", Eng. Struct., 28, 240-254.

Chong, M. S., Perry, A. E. and Cantwell, B. J. (1990), "A general classification of three-dimensional flow field", Phys. Fluids A: Fluid Dynamics, 2765.

Cucitore, R., Quadrio, M. and Baron, A. (1999), "On the effectiveness and limitations of local criteria for the identification of a vortex", European J. Fluid Mech. B/Fluids, 18(2), 261-282.

Fiedler, H. E. (1988), "Coherent structures in turbulent flows", Progress in Aerospace Sci., 25, 3, 231-269.

Hunt, J. C. R., Wray, A. A. and Moin, P. (1988), "Eddies, stream and convergence zones in turbulent flow", Center for Turbulence Research Report CTR-S88, 193.

Jeong, J. and Hussain, A. K. M. F. (1995), “On the identification of a vortex”, J. Fluid Mech., 285, 69-94.

Larsen, G., Bierbooms, W. and Hansen, K. (2003), "Mean gust shapes", Risoe National Laboratory, Denmark, Report 1133 EN.

McNaughton, K. G. (2004), "Turbulence structure of the unstable atmospheric surface layer and transition to the outer layer", Boundary Layer Meteorology, 112, 199-221.

Oseguera, R. and Bowles, R. (1988), "A simple, analytic 3-dimensional downburst model based on boundary layer stagnation flow", NASA TM, 100632.

Ovenden, N. C. and Smith, F. T. (2003), "On generation of horseshoe vortices by corrugated surfaces, surface roughnesses or pipe bends", J. Eng. Math., 45, 5-20.

Richards, P. J., Fong, S. and Hoxey, R. P. (1997), "Anisotropic turbulence in the atmospheric surface layer", $J$. Wind Eng. Ind. Aerodyn., 69-71, 903-913.

Robinson, S. K. (1991), "Coherent motions in the turbulent boundary layer", Ann. Rev. Fluid Mech., 23, 601639.

Skote, M., Haritonidis, J. H., and Henningson, D. S. (2002), "Varicose instabilities in turbulent boundary layers", Phys. Fluids, 14(7), 2309-2323.

Sterling, M., Baker, C. J. and Hoxey, R. P. (2003), "Short term unsteady wind loading on a low-rise building", Wind and Struct., 6(5), 403-418.

Sterling, M., Baker, C. J., Quinn, A. D. and Hoxey, R. P. (2005), "Pressure and velocity fluctuations in the atmospheric boundary layer", Wind and Struct., 8(1), 13-34.

Sterling, M., Baker, C. J., Richards, P. J., Hoxey, R. P. and Quinn, A. D. (2006), “An investigation of the wind statistics and extreme gusts at a rural site", Wind and Struct., 9(3), 193-215.

Willmarth, W. W. and Lu, S. S. (1972), "Structure of the Reynolds stress near the wall”, J. Fluid Mech., 55, 6592.

Zhou, J., Adrian, R. J. and Balachandar, S. (1999), "Mechanisms for generating coherent packets of hairpin vortices in channel flow", J. Fluid Mech., 387, 353-396.

$J H$ 